\title{
Pulmonary Embolism Response Team activation during the COVID-19 pandemic in a New York City Academic Hospital: a retrospective cohort analysis
}

\author{
Benjamin Kwok ${ }^{1}$. Shari B. Brosnahan ${ }^{1}$. Nancy E. Amoroso ${ }^{1} \cdot$ Ronald M. Goldenberg $^{1}$ • Brooke Heyman ${ }^{1}$. \\ James M. Horowitz ${ }^{2}$. Catherine Jamin ${ }^{3} \cdot$ Akhilesh K. Sista $^{4} \cdot$ Deane E. Smith ${ }^{5} \cdot$ Eugene Yuriditsky $^{2}$. \\ Thomas S. Maldonado ${ }^{6}$
}

Published online: 10 September 2020

(c) Springer Science+Business Media, LLC, part of Springer Nature 2020

\begin{abstract}
Coronavirus disease 2019 (COVID-19) is associated with increased rates of deep vein thrombosis (DVT) and pulmonary embolism (PE). Pulmonary Embolism Response Teams (PERT) have previously been associated with improved outcomes. We aimed to investigate whether PERT utilization, recommendations, and outcomes for patients diagnosed with acute PE changed during the COVID-19 pandemic. This is a retrospective cohort study of all adult patients with acute PE who received care at an academic hospital system in New York City between March 1st and April 30th, 2020. These patients were compared against historic controls between March 1st and April 30th, 2019. PE severity, PERT utilization, initial management, PERT recommendations, and outcomes were compared. There were more cases of PE during the pandemic (82 vs. 59), but less PERT activations $(26.8 \%$ vs. $64.4 \%, p<0.001)$ despite similar markers of PE severity. PERT recommendations were similar before and during the pandemic; anticoagulation was most recommended ( $89.5 \%$ vs. $86.4 \%, p=0.70)$. During the pandemic, those with PERT activations were more likely to be female (63.6\% vs. 31.7\%, $p=0.01)$, have a history of DVT/PE $(22.7 \%$ vs. $1.7 \%, p=0.01)$, and to be SARS-CoV-2 PCR negative ( $68.2 \%$ vs. $38.3 \% p=0.02)$. PERT activation during the pandemic is associated with decreased length of stay $(7.7 \pm 7.7$ vs. $13.2 \pm 12.7$ days, $p=0.02)$. PERT utilization decreased during the COVID-19 pandemic and its activation was associated with different biases. PERT recommendations and outcomes were similar before and during the pandemic, and led to decreased length of stay during the pandemic.
\end{abstract}

Keywords Venous thromboembolism · Pulmonary embolism · Pulmonary Embolism Response Team · COVID-19 · SARS$\mathrm{CoV}-2$

Abbreviations
BNP
CDT

COVID-19

Brain natriuretic peptide

Catheter-directed therapy

Coronavirus disease 2019

Electronic supplementary material The online version of this article (https://doi.org/10.1007/s11239-020-02264-8) contains supplementary material, which is available to authorized users.

Benjamin Kwok

benjamin.kwok@nyulangone.org

1 Division of Pulmonary, Critical Care and Sleep Medicine, New York University Langone Health, New York, NY, USA

2 Leon H. Charney Division of Cardiology, New York University Langone Health, New York, NY, USA

3 Ronald O. Perelman Department of Emergency Medicine, New York University Langone Health, New York, NY, USA

$\begin{array}{ll}\text { COVID era } & \text { March 1, 2020-April 30, 2020 } \\ \text { CT-PE } & \begin{array}{l}\text { Computed tomography-pulmonary } \\ \text { embolism }\end{array} \\ \text { DVT } & \text { Deep vein thrombosis } \\ \text { ESC } & \text { European Society of Cardiology } \\ \text { IVC } & \text { Inferior vena cava } \\ \text { LMWH } & \text { Low molecular weight heparin }\end{array}$

4 Division of Vascular and Interventional Radiology, New York University Langone Health, New York, NY, USA

5 Department of Cardiothoracic Surgery, New York University Langone Health, New York, NY, USA

6 Division of Vascular and Endovascular Surgery, New York University Langone Health, New York, NY, USA 


$\begin{array}{ll}\text { LOS } & \text { Length of stay } \\ \text { PE } & \text { Pulmonary embolism } \\ \text { PERT } & \text { Pulmonary Embolism Response Team } \\ \text { PESI } & \text { Pulmonary Embolism Severity Index } \\ \text { pre-COVID era } & \text { March 1, 2019-April 30, 2019 } \\ \text { RT-PCR } & \begin{array}{l}\text { Reverse transcription-polymerase chain } \\ \text { reaction }\end{array} \\ \text { RV } & \begin{array}{l}\text { Right ventricle } \\ \text { SARS-CoV-2 }\end{array} \\ \begin{array}{l}\text { Severe acute respiratory syndrome coro- } \\ \text { navirus 2 }\end{array} \\ \text { UFH } & \text { Unfractionated heparin } \\ \text { VTE } & \text { Venous thromboembolism }\end{array}$

\section{Highlights}

- We reviewed patients with acute pulmonary embolism (PE) at our institution during the COVID-19 pandemic from March 1st to April 30th, 2020.

- Of 82 cases of PE during the COVID-19 pandemic, 22 (26.8\%) resulted in a Pulmonary Embolism Response Team (PERT) activation.

- PERT activations decreased during the COVID-19 pandemic.

- PERT activations were associated with a shorter length of stay.

\section{Introduction}

Mounting evidence for the associations between coronavirus disease 2019 (COVID-19), hypercoagulability, and mortality from thrombus formation have led to debate over the prevention and treatment of thrombi in this population [1]. While some institutions have used higher-intensity dosing of venous thromboembolism (VTE) chemoprophylaxis and modified treatment algorithms, there are no published guidelines recommending this change in therapy $[2,3]$.

Outside of COVID-19, pulmonary embolisms (PE) and VTE are common causes of cardiovascular morbidity and mortality, with high-risk (i.e. massive) PE having mortality rates of 25-65\% [4-8]. Multidisciplinary Pulmonary Embolism Response Teams (PERT) help standardize and individualize complex medical decision-making. PERTs help physicians navigate the numerous treatment modalities including systemic thrombolysis, catheter-direct therapies (CDT), surgical embolectomy, and placement of inferior vena cava (IVC) filters. Evidence suggests PERTs may improve outcomes by reducing non-major bleeding, time to therapeutic anticoagulation, and mortality rates [7].

There is increasing focus of the differences between COVID-19 and non-COVID-19 VTE including incidence and changes in inflammatory makers (e.g. fibrinogen and D-dimer), coagulation panel, platelets, and thromboelastography parameters [9-11]. The COVID-19 pandemic has challenged healthcare resources and changed the practice of medicine. The effects have been widespread and have included issues such as imaging and bed availability, scarcity of blood products, and provider overextension [12-15]. Reports of healthcare providers practicing anecdotal medicine based on theories and conjecture have surfaced, leading to calls to practice "rational" care and wait for additional evidence and clinical trials before shifting patient care strategies $[2,16,17]$. PERTs provide high quality evidencedbased care in a disease where risks and benefits vary from patient to patient [7]. The impact of the COVID-19 pandemic on PERT-related outcomes is currently unknown given the lack of understanding of COVID-19-related thrombosis and evidence-based management guidelines. Presently, there are no studies examining the usage of PERT during the COVID-19 pandemic. Our study aims to see if these stressors: strain on resource allocation, seemingly higherthan expected incidence of VTE, and off-label use of anticoagulation and thrombolysis, have changed PERT activations and recommendations. Our study aims to compare PE as well as PERT activations between the pre-COVID and the COVID eras.

\section{Methods}

We performed a retrospective cohort analysis of all patients greater than or equal to 18-years-old who received emergency department or inpatient care at an academic hospital in New York City between March 1st and April 30th, 2020 (COVID era) and were found to have radiographically confirmed PE. Positive imaging studies were found via our institution's internal Venous Thromboembolism Center quality improvement database, which logs all images confirming VTE and all PERT activations. March 1st and April 30th, 2020 were chosen because of peak COVID-19-related emergency department visits. Historical controls of patients were obtained same period one-year prior (March 1st to April 30th, 2019, pre-COVID era). The Institutional Review Board at New York University Langone Health Center approved this study (\#20-00501). There was no funding source for this study.

Patient charts were individually reviewed to gather variables including patient demographics, comorbidities (presence of hypertension, heart failure, diabetes mellitus, chronic lung disease, chronic kidney disease, malignancy, prior stroke, prior myocardial infarction, prior DVT or PE), and PERT activation. Parameters required to calculate a Pulmonary Embolism Severity Index (PESI) and timing of 
diagnosis and PERT activation were noted. Presence of right ventricular (RV) dysfunction was identified if revealed by imaging (echocardiogram and/or CT-PE) or by elevated cardiac biomarkers including troponin or brain natriuretic peptide (BNP), as defined by our laboratory-specific cut-offs. PE risk was stratified into low, intermediate-low, intermediatehigh, and high risk categories according to the European Society of Cardiology (ESC) 2019 guidelines [8]. Additional labs included severe acute respiratory disease coronavirus 2 (SARS-CoV-2) real time reverse transcription polymerase chain reaction (RT-PCR) for COVID-19. Initial management strategies (within $48 \mathrm{~h}$ of diagnosis) and PERT recommendations were recorded. Outcomes including death prior to discharge, bleeding, length of stay (LOS), and readmission within 30 days were compared.

Data was exported from REDCap to IBM SPSS Statistics (version 25) for analysis. Continuous variables are presented as means with standard deviations and compared using Student's unpaired $t$ test. Categorical variables are presented as frequency and compared using Pearson's chi-squared or Fischer's exact test. Significance was assessed using twotailed t-test with $p<0.05$ considered significant.

\section{Results}

During the COVID era, there were 82 patients with PE who received emergency department or inpatient care; 22 of these patients had PERT activations. Of the 82 patients, 44 patients were COVID-positive and 7 (16\%) of COVIDpositive patients with VTE had PERT activations. In the pre-COVID era, we identified 59 patients with emergency department visits or inpatient hospitalizations who had PE; 38 of these patients had PERT activations. We have three unique comparisons that characterize PE and PERT utilization: (1) all PEs before and during COVID era, (2) PERT and non-PERT activations pre-COVID era and COVID era, and (3) PERT and non-PERT activations during COVID era.

\section{Comparison of PE pre-COVID era and COVID era}

Patients with PE in the pre-COVID era were older $(64 \pm 15.3$ vs. $58.1 \pm 16.2$ years, $p=0.03$ ), more likely to have malignancy $(30.5 \%$ vs. $14.6 \%, p=0.02)$, and more likely to be on anticoagulation prior to admission $(15.3 \%$ vs. $4.9 \%, p=0.04)$ than those with PE during the COVID era. Otherwise, there were no statistically significant differences in gender, BMI, residence prior to encounter, and rates of tobacco and heavy alcohol use, and comorbidities (Supplemental Table 1).

Patients in the pre-COVID era were diagnosed with PE earlier in their hospitalization $(0.97 \pm 3.2$ vs. $4.21 \pm 7.5$ days, $p=0.001)$. While there was an increase in cases of PE during the COVID-19 pandemic compared to the pre-COVID era, there was a decrease in proportion of PERT activations ( $26.8 \%$ vs. $64.4 \%, p<0.001$ ). The time of PERT activation relative to time of diagnosis was not statistically different $(0.23 \pm 0.48$ vs. $0.55 \pm 1.9$ days, $p=0.32)$. The presence of RV strain by imaging and biomarkers (BNP and troponin), PESI, and risk stratification by ESC guidelines were similar. The majority of cases were of the intermediate-low risk category during both time periods.

Most patients in both eras were initially treated with anticoagulation alone $(88.1 \%$ vs. $93.9 \%, p=0.23)$. In the pre-COVID era, more patients received advanced therapies, specifically catheter-directed thrombolysis $(6.8 \%$ vs. $0 \%$, $p=0.03)$ and catheter embolectomy ( $8.5 \%$ vs. $0 \%, p=0.01)$ (Table 1). Initial anticoagulation choice differed between eras, with unfractionated heparin (UFH) being used more frequently in the pre-COVID era $(69.5 \%$ vs. $50 \%, p=0.02)$ and low molecular weight heparin (LMWH) used more often in the COVID era (40.2 vs. $8.5 \%, p<0.001)$. Patients had shorter LOS in the pre-COVID era, especially in the intermediate-low risk group ( $7.2 \pm 10.7$ vs. $15.2 \pm 13.7$ days, $p=0.004)$, but were more likely to be re-admitted within 30 days $(20.3 \%$ vs. $8.5 \% p=0.04)$. There was no difference in deaths prior to discharge and bleeding events.

\section{PERT activations before and during COVID era}

During the pre-COVID era, 38 of the 59 new PE cases (64.4\%) had a PERT activation compared to 22 of 82 (26.8\%) during the COVID era. There were higher levels of serum brain natriuretic peptide (BNP) during the preCOVID era $(545 \pm 1054$ vs. $118.2 \pm 200 \mathrm{pg} / \mathrm{mL}, p=0.04)$. Otherwise, there were no significant differences in risk factors, severity indices, or PE classification (Table 2). PERT recommendations were similar, with a majority of patients being managed with anticoagulation alone $(89.5 \%$ vs. $86.4 \%$, $p=0.70$ ); UFH was the most frequent choice of initial anticoagulant $(78.9 \%$ vs $68.2 \%, p=0.35)$.

Patients without PERT activations during the pre-COVID era were less likely to be on LMWH $(9.5 \%$ vs. $48.3 \%$, $p=0.002$ ), more likely to be on a direct oral anticoagulant ( $28.6 \%$ vs. $6.7 \%, p=0.01)$, and had a shorter LOS $(5.1 \pm 5.7$ vs. $13.2 \pm 12.7$ days, $p<0.001$ ), especially for those with intermediate-low risk PE. There was also a trend towards less bleeding ( $4.8 \%$ vs. $25 \%, p=0.057$ ) for those without PERT activation in the pre-COVID era.

\section{PERT activations versus non-PERT activations in COVID era patients}

There were 22 PE cases with PERT activations and 60 without during COVID era (Table 3). Patients with PERT 
Table 1 Comparison of PE in the Pre-COVID and COVID era

\begin{tabular}{|c|c|c|c|}
\hline & $\begin{array}{l}\text { Pre-COVID era } \\
n=59\end{array}$ & $\begin{array}{l}\text { COVID-era } \\
n=82\end{array}$ & $p$ value \\
\hline \multicolumn{4}{|l|}{ Diagnosis } \\
\hline DVT, n (\%) & $28(47.5)$ & $30(36.6)$ & 0.2 \\
\hline Hospital day of diagnosis, mean (SD) & $0.97 \pm 3.2$ & $4.21 \pm 7.5$ & $0.001^{\mathrm{a}}$ \\
\hline Hospital day of PERT activation, mean (SD) & $0.65 \pm 1.3$ & $1.68 \pm 3.8$ & 0.23 \\
\hline Delay of PERT activation, day, mean (SD) & $0.23 \pm 0.48$ & $0.55 \pm 1.9$ & 0.32 \\
\hline PERT activated? & $38(64.4)$ & $22(26.8)$ & $<0.001^{\mathrm{a}}$ \\
\hline \multicolumn{4}{|l|}{ Initial PERT recommendation or management, $\mathrm{n}(\%)$} \\
\hline Anticoagulation, only & $52(88.1)$ & $77(93.9)$ & 0.23 \\
\hline Thrombolysis (systemic) & $1(1.7)$ & $1(1.2)$ & 1.00 \\
\hline Thrombolysis (catheter-directed) & $4(6.8)$ & 0 & $0.03^{\mathrm{a}}$ \\
\hline IVC filter & $3(5.1)$ & $3(3.7)$ & 0.70 \\
\hline Embolectomy (surgical) & 0 & 0 & - \\
\hline Embolectomy (catheter) & $5(8.5)$ & 0 & $0.01^{\mathrm{a}}$ \\
\hline \multicolumn{4}{|l|}{ Initial anticoagulant choice } \\
\hline Unfractionated heparin & $41(69.5)$ & $41(50)$ & $0.02^{\mathrm{a}}$ \\
\hline Low molecular weight heparin & $5(8.5)$ & $33(40.2)$ & $<0.001^{\mathrm{a}}$ \\
\hline Oral direct anti-Xa inhibitor & $9(15.3)$ & $6(7.3)$ & 0.13 \\
\hline Other & 0 & $1(1.2)$ & 1.00 \\
\hline None & $4(6.8)$ & $1(1.2)$ & 0.16 \\
\hline \multicolumn{4}{|l|}{ Outcomes } \\
\hline Death prior to discharge, $\mathrm{n}(\%)$ & $5(8.5)$ & $10(12.2)$ & 0.48 \\
\hline Length of stay, days, mean (SD) & $6.5 \pm 9.2$ & $11.7 \pm 11.7$ & $0.004^{\mathrm{a}}$ \\
\hline Low risk & $4.5 \pm 7.9$ & $7.2 \pm 8.2$ & 0.39 \\
\hline Intermediate-low risk & $7.2 \pm 10.7$ & $15.2 \pm 13.7$ & $0.004^{\mathrm{a}}$ \\
\hline Intermediate-high risk & $5.6 \pm 3.1$ & $8.5 \pm 5.5$ & 0.16 \\
\hline High risk & $8.3 \pm 6.5$ & - & - \\
\hline Readmission within 30-days, $\mathrm{n}(\%)$ & $12(20.3)$ & $7(8.5)$ & $0.04^{\mathrm{a}}$ \\
\hline Bleeding, n (\%) & 7 (11.9) & $18(22)$ & 0.12 \\
\hline
\end{tabular}

Continuous variables are presented as mean (standard deviation, SD); comparisons are performed using unpaired t-test. Categorical variables are presented as frequency; comparisons are performed using Pearson's chi-squared or Fischer's exact test. IVC, inferior vena cava

${ }^{a} p<0.05$ calculated by Pearson chi-squared test or Student's unpaired t test activations were more likely to be female (63.6\% vs. $31.7 \%$, $p=0.01)$, have a history of DVT/PE $(22.7 \%$ vs. $1.7 \%$, $p=0.01$ ), and to be taking anticoagulation prior to admission $(13.6 \%$ vs. $1.7 \%, p=0.057)$. They were also more likely to be SARS-CoV-2 RT-PCR negative $(68.2 \%$ vs. $38.3 \%$ $p=0.02)$, to be diagnosed with a concurrent DVT (77.3\% vs. $21.7 \%, p<0.001)$, and to be diagnosed earlier during their hospitalization $(1.1 \pm 2.3$ vs. $5.3 \pm 8.4$ days, $p=0.001)$. In terms of PE severity, those with PERT activations had more incidences of RV strain on imaging $(54.5 \%$ vs. $18.3 \%$, $p=0.001)$, but lower PESI $(83.1 \pm 26.0$ vs. $103.6 \pm 35.7$, $p=0.01$ ). There was an increased proportion of intermediate-high risk PE in those with PERT activations, but this did not reach statistical significance $(28.6 \%$ vs. $13.5 \%, p=0.31$ ). Overall LOS was shorter with PERT activations $(7.7 \pm 7.7$ vs. $12.2 \pm 12.7$ days, $p=0.02$ ).

\section{Discussion}

We found that PERT activations decreased during the COVID-19 pandemic despite similar markers of risk and PE categories. The pandemic did not change PERT recommendations but did change how non-PERT PE cases were managed. This was the major hypothesis of our study, as PERT members had anecdotally felt that physicians were changing their normal medical management during the COVID era.

COVID era patients with PE were younger than prior PE patients with less known PE risk factors including malignancy. PE in the COVID era patients occurred later in patient's hospital course than pre-COVID era patients. This is interesting because all COVID era patients were placed on VTE chemoprophylaxis, unless otherwise contraindicated. 
Table 2 Comparison of PERT activations before and during COVID-19 pandemic

\begin{tabular}{|c|c|c|c|c|c|c|}
\hline & \multicolumn{3}{|l|}{ PERT activations } & \multicolumn{3}{|c|}{ No PERT activations } \\
\hline & $\begin{array}{l}\text { Pre-COVID era } \\
n=38\end{array}$ & $\begin{array}{l}\text { COVID-era } \\
n=22\end{array}$ & $p$ value & $\begin{array}{l}\text { Pre-COVID era } \\
n=21\end{array}$ & $\begin{array}{l}\text { COVID-era } \\
n=60\end{array}$ & $p$ value \\
\hline \multicolumn{7}{|l|}{ Diagnosis } \\
\hline DVT, n (\%) & $20(52.6)$ & $17(77.3)$ & 0.06 & $8(38.1)$ & $13(21.7)$ & 0.14 \\
\hline Hospital day of diagnosis, mean (SD) & $0.45 \pm 1.33$ & $1.1 \pm 2.3$ & 0.20 & $1.9 \pm 4.9$ & $5.3 \pm 8.4$ & $0.03^{\mathrm{a}}$ \\
\hline Hospital day of PERT activation, mean(SD) & $0.68 \pm 1.34$ & $1.7 \pm 3.8$ & 0.24 & - & - & - \\
\hline Delay of PERT activation, day, mean (SD) & $0.24 \pm 0.49$ & $0.55 \pm 1.9$ & 0.35 & - & - & - \\
\hline \multicolumn{7}{|l|}{ Severity indices } \\
\hline Troponin I, admission (ng/mL) & $0.119 \pm 0.206$ & $0.281 \pm 0.670$ & 0.32 & $0.068 \pm 0.143$ & $0.234 \pm 0.707$ & 0.37 \\
\hline $\mathrm{BNP}$, admission $(\mathrm{pg} / \mathrm{mL})$ & $545 \pm 1054$ & $118.2 \pm 200.0$ & $0.04^{\mathrm{a}}$ & $125 \pm 139$ & $168 \pm 350.2$ & 0.69 \\
\hline $\mathrm{RV}$ strain on imaging, $\mathrm{n}(\%)$ & $14(36.8)$ & $12(54.5)$ & 0.18 & $3(14.3)$ & $11(18.3)$ & 1.0 \\
\hline Hypotension, n (\%) & $3(7.9)$ & 0 & 0.29 & 0 & $1(1.7)$ & 1.0 \\
\hline PESI, mean (SD) & $90.5 \pm 26.1$ & $83.1 \pm 26.0$ & 0.30 & $94.2 \pm 34.8$ & $103.6 \pm 35.7$ & 0.30 \\
\hline PE severity risk, n (\%) & & & 0.10 & & & \\
\hline Low & $5(13.2)$ & $6(27.3)$ & 0.19 & $5(23.8)$ & $17(28.3)$ & 0.69 \\
\hline Intermediate-low & $23(60.5)$ & $9(40.9)$ & 0.18 & $14(66.7)$ & $35(58.3)$ & 0.50 \\
\hline Intermediate-high & $7(18.4)$ & $7(31.8)$ & 0.34 & $2(9.5)$ & $8(13.3)$ & 1.0 \\
\hline High & $3(7.9)$ & 0 & 0.29 & 0 & 0 & - \\
\hline \multicolumn{7}{|l|}{ Initial PERT recommendation or management $\mathrm{n}(\%)$} \\
\hline Anticoagulation, only & $34(89.5)$ & $19(86.4)$ & 0.70 & $18(85.7)$ & $58(96.7)$ & 0.11 \\
\hline Thrombolysis (systemic) & 1 & 0 & 1.0 & 0 & 1 & 1.0 \\
\hline Thrombolysis (catheter-directed) & 2 & 0 & 0.53 & 2 & 0 & 0.07 \\
\hline IVC filter & 3 & 2 & 1.0 & 0 & 0 & - \\
\hline Embolectomy (surgical) & 0 & 0 & - & 0 & 0 & - \\
\hline Embolectomy (catheter) & 3 & 0 & 0.29 & 2 & 0 & 0.07 \\
\hline \multicolumn{7}{|l|}{ Initial anticoagulant choice } \\
\hline Unfractionated heparin & $30(78.9)$ & $15(68.2)$ & 0.35 & $11(52.4)$ & $26(43.3)$ & 0.47 \\
\hline Low molecular weight heparin & $3(7.9)$ & $4(18.2)$ & 0.41 & $2(9.5)$ & $29(48.3)$ & $0.002^{\mathrm{a}}$ \\
\hline Oral direct anti-Xa inhibitor & $3(7.9)$ & $2(9.1)$ & 1.0 & $6(28.6)$ & $4(6.7)$ & $0.01^{\mathrm{a}}$ \\
\hline Other & 0 & $1(4.5)$ & 0.37 & 0 & 0 & - \\
\hline None & $2(5.3)$ & 0 & 0.53 & $2(9.5)$ & $1(1.7)$ & 0.16 \\
\hline \multicolumn{7}{|l|}{ Outcomes } \\
\hline Death prior to discharge, $\mathrm{n}(\%)$ & $2(5.3)$ & $1(4.5)$ & 1.0 & $3(14.3)$ & $9(15)$ & 1.0 \\
\hline Length of stay, days, mean (SD) & $7.3 \pm 10.6$ & $7.7 \pm 7.7$ & 0.90 & $5.1 \pm 5.7$ & $13.2 \pm 12.7$ & $<0.001^{\circ}$ \\
\hline Low risk & $1.6 \pm 0.9$ & $4.0 \pm 3.2$ & 0.14 & $7.4 \pm 10.9$ & $8.3 \pm 9.2$ & 0.86 \\
\hline Intermediate-low risk & $9.1 \pm 13.1$ & $10.6 \pm 19.8$ & 0.77 & $3.9 \pm 2.9$ & $16.4 \pm 14.3$ & $<0.001$ \\
\hline Intermediate-high risk & $5.1 \pm 3.2$ & $7.1 \pm 4.2$ & 0.34 & $7.0 \pm 2.8$ & $9.6 \pm 6.5$ & 0.61 \\
\hline High risk & $8.33 \pm 6.43$ & - & - & - & - & - \\
\hline Readmission within 30 -days, $\mathrm{n}(\%)$ & $10(26.3)$ & $2(9.1)$ & 0.18 & $2(9.5)$ & $5(8.3)$ & 1.0 \\
\hline Bleeding, $\mathrm{n}(\%)$ & $6(15.8)$ & $3(13.6)$ & 1.0 & $1(4.8)$ & $15(25)$ & 0.057 \\
\hline
\end{tabular}

Upper limit of normal (ULN) for brain natriuretic peptide (BNP) is $100 \mathrm{pg} / \mathrm{mL}$. ULN for troponin is $0.04 \mathrm{ng} / \mathrm{mL}$. $R V$ right ventricle, $I V C$ inferior vena cava. Continuous variables are presented as mean (standard deviation, SD); comparisons are performed using unpaired t-test. Categorical variables are presented as frequency; comparisons are performed using Pearson's chi-squared or Fischer's exact test

${ }^{a} p<0.05$ calculated by Pearson chi-squared test or Student's unpaired t test

This supports that COVID patients may still be at increased risk to develop clots despite thromboembolism prophylaxis.
Our data corroborates that there appears to be a higher incidence of PE in patients with COVID than those without COVID. However, limitations in securing a radiographic 
Table 3 Comparison of PERT and no PERT activation in patients during COVID-19 pandemic

\begin{tabular}{|c|c|c|c|}
\hline & \multicolumn{3}{|l|}{ COVID-era } \\
\hline & $\begin{array}{l}\text { PERT activation } \\
n=22\end{array}$ & $\begin{array}{l}\text { No PERT activation } \\
n=60\end{array}$ & $p$ value \\
\hline \multicolumn{4}{|l|}{ Diagnosis } \\
\hline DVT, n (\%) & $17(77.3)$ & $13(21.7)$ & $<0.001^{\mathrm{a}}$ \\
\hline Hospital day of diagnosis, mean (SD) & $1.1 \pm 2.3$ & $5.3 \pm 8.4$ & $0.001^{\mathrm{a}}$ \\
\hline Hospital day of PERT activation, mean (SD) & $1.7 \pm 3.8$ & - & - \\
\hline Delay of PERT activation, day, mean (SD) & $0.55 \pm 1.9$ & - & - \\
\hline SARS-CoV-2 RT-PCR Positive & $7(31.8)$ & $37(61.7)$ & $0.02^{\mathrm{a}}$ \\
\hline \multicolumn{4}{|l|}{ Severity Indices } \\
\hline Troponin I, admission (ng/mL) & $0.281 \pm 0.670$ & $0.234 \pm 0.707$ & 0.8 \\
\hline $\mathrm{BNP}$, admission $(\mathrm{pg} / \mathrm{mL})$ & $118.2 \pm 200.0$ & $168 \pm 350.2$ & 0.6 \\
\hline RV strain on imaging, $\mathrm{n}(\%)$ & $12(54.5)$ & $11(18.3)$ & $0.001^{\mathrm{a}}$ \\
\hline Hypotension, n (\%) & 0 & $1(1.7)$ & 1.0 \\
\hline PESI, mean (SD) & $83.1 \pm 26.0$ & $103.6 \pm 35.7$ & $0.02^{\mathrm{a}}$ \\
\hline PE severity risk, n (\%) & & & 0.16 \\
\hline Low & $6(27.3)$ & $17(28.3)$ & 0.93 \\
\hline Intermediate-low & $9(40.9)$ & $35(58.3)$ & 0.16 \\
\hline Intermediate-high & $7(31.8)$ & $8(13.3)$ & 0.1 \\
\hline High & 0 & 0 & - \\
\hline \multicolumn{4}{|c|}{ Initial PERT recommendation or management, $\mathrm{n}(\%)$} \\
\hline Anticoagulation, only & $19(86.4)$ & $58(96.7)$ & 0.12 \\
\hline Thrombolysis (systemic) & 0 & 1 & 1.0 \\
\hline Thrombolysis (catheter-directed) & 0 & 0 & - \\
\hline IVC filter & 2 & 0 & 0.07 \\
\hline Embolectomy (surgical) & 0 & 0 & - \\
\hline Embolectomy (catheter) & 0 & 0 & - \\
\hline \multicolumn{4}{|l|}{ Initial anticoagulant choice } \\
\hline Unfractionated heparin & $15(68.2)$ & $26(43.3)$ & $0.046^{\mathrm{a}}$ \\
\hline Low molecular weight heparin & $4(18.2)$ & $29(48.3)$ & $0.01^{\mathrm{a}}$ \\
\hline Oral direct anti-Xa inhibitor & $2(9.1)$ & $4(6.7)$ & 0.66 \\
\hline Other & $1(4.5)$ & 0 & 0.27 \\
\hline None & 0 & $1(1.7)$ & 1.0 \\
\hline \multicolumn{4}{|l|}{ Outcomes } \\
\hline Death prior to discharge, $\mathrm{n}(\%)$ & $1(4.5)$ & $9(15)$ & 0.28 \\
\hline Length of stay, days, mean (SD) & $7.7 \pm 7.7$ & $13.2 \pm 12.7$ & $0.02^{\mathrm{a}}$ \\
\hline Low risk & $4.0 \pm 3.2$ & $8.3 \pm 9.2$ & 0.28 \\
\hline Intermediate-low risk & $10.6 \pm 19.8$ & $16.4 \pm 14.3$ & 0.26 \\
\hline Intermediate-high risk & $7.1 \pm 4.2$ & $9.6 \pm 6.5$ & 0.41 \\
\hline High risk & - & - & \\
\hline Readmission within 30-days, n (\%) & $2(9.1)$ & $5(8.3)$ & 1.0 \\
\hline Bleeding, $\mathrm{n}(\%)$ & $3(13.6)$ & $15(25)$ & 0.37 \\
\hline
\end{tabular}

Upper limit of normal (ULN) for brain natriuretic peptide (BNP) is $100 \mathrm{pg} / \mathrm{mL}$. ULN for troponin is $0.04 \mathrm{ng} / \mathrm{mL}$. SARS-CoV-2 - severe acute respiratory syndrome coronavirus 2 . RT-PCR, reverse transcription-polymerase chain reaction. RV, right ventricle. IVC, inferior vena cava. Continuous variables are presented as mean (standard deviation, SD); comparisons are performed using unpaired t-test. Categorical variables are presented as frequency; comparisons are performed using Pearson's chi-squared or Fischer's exact test

${ }^{\mathrm{a}} p<0.05$ calculated by Pearson chi-squared test or Student's unpaired t test 


\section{COVID-19 and Pulmonary Embolism Response Team (PERT)}

\section{Does your patient have COVID-19 with confirmed or suspected PE and any of:}

\section{Tachycardia \\ Acutely worsening dyspnea \\ Hemoptysis \\ Moderate to severe chest pain \\ Echo with right ventricular dilation \\ CT-PE with signs of right heart strain \\ Saddle embolism or significant clot burden \\ Serum troponin above upper limit of normal}

\section{Was your patient started on empiric anticoagulation for a presumed PE (no image confirmation) and is now nearing discharge?}

If yes to either, then consult PERT.

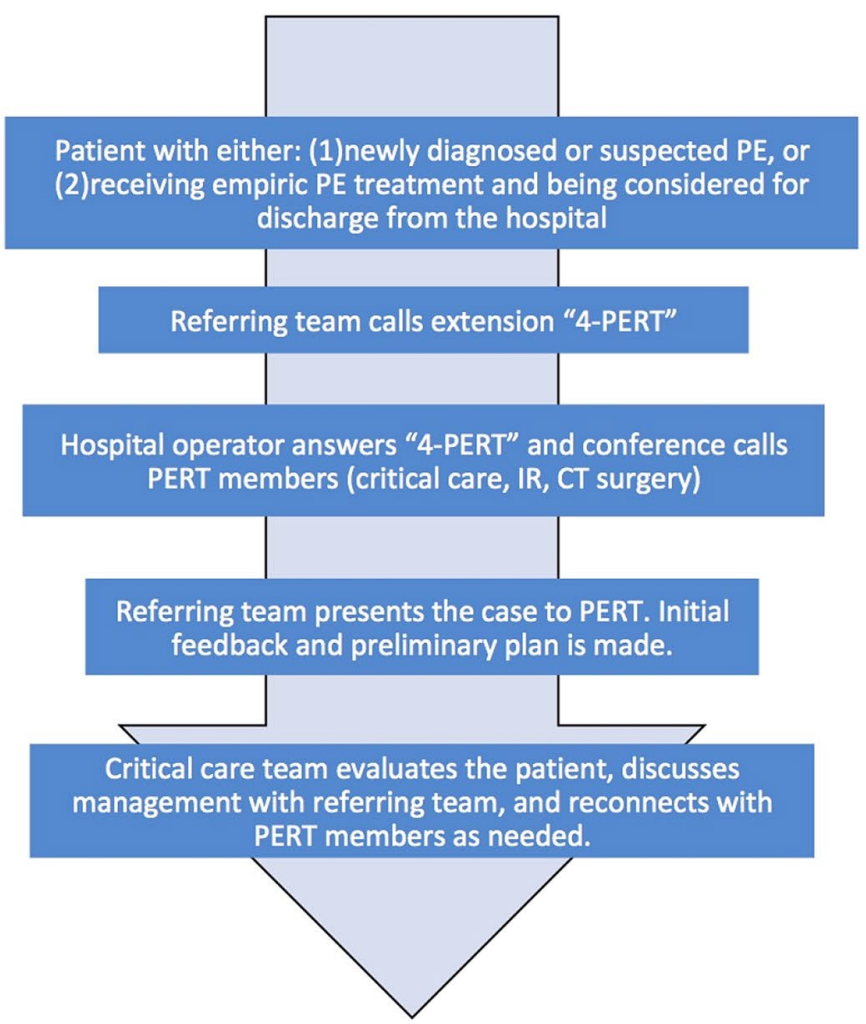

Fig. 1 PERT alert work flow and COVID-19 infection. CT surgery cardiothoracic surgery, CT-PE computed tomography-pulmonary embolism, $P E$ pulmonary embolism, PERT Pulmonary Embolism Response Team, IR interventional radiology

diagnosis in a majority of patients with COVID due to resource allocation may skew these results. Unexpectedly, there was nearly a $40 \%$ decrease in PERT activations during the COVID era. This highlights that the PERT activation is subjective and providers can choose to practice without using the expertise of PERT. In fact, PERT activations during COVID era were predominately on female patients, those with a history of DVT/PE, those on anticoagulants prior to hospital encounter, and those who were SARS-CoV-2 RTPCR negative. These risk factors and anticoagulant failure may have motivated providers to activate PERT. Those who were SARS-CoV-2 RT-PCR positive were less likely to have a PERT activation, another suggestion that physicians had bias when caring for these patients.

The major driver for the decrease in PERT activations, specifically on COVID-positive patients, is not explained. It is possible that there were more "critical care"-trained physicians attending during this time who did not feel extra input was needed, that there was little role for advanced therapy during this period of contagion, that there was little evidence to guide treatment for COVID-positive patients, or that the chaos of the pandemic changed physician's typical practice. PERT recommendations were consistent before and throughout the COVID era. However, there were trends towards more use of LMWH and less CDT, though neither reached statistical significance. In cases where PERT was activated, outcomes were similar between time periods. It should be noted the PERT activations in the COVID era, were much more likely to occur on SARS-CoV-2 RT-PCR negative patients, which further supports that the presence of COVID-positivity was the main driver for the deviation in standard care rather than any other the other hypotheses listed.

During the COVID era, differences in patient characteristics between PERT and non-PERT activation gives another glimpse into the factors that motivate physicians to active PERT. While patients were well-matched by age, gender, BMI, and co-morbidities, those with PERT activation were more likely to have concurrent DVT and RV strain by imaging. The discordance between these findings and the lower PESI and lack of statistically significant differences in PE risk (by ESC guidelines) highlights the heterogeneity and difficulty in classifying submassive PE. PERT activation correlated with almost a 50\% reduction in LOS and 
trend towards fewer bleeding events. These are important outcomes because they may potentially relieve strain on important resources. Reduced LOS increases bed availability and the ability to accommodate increases in patient volume. Fewer bleeding events allows for conservation of scarce blood products [18]. These positive outcomes by PERT occurred without a complete understanding of the pathophysiology of COVID-19 or evidence-based management guidelines. Thus, the use of PERTs should be encouraged during future pandemics to optimize resource allocation.

There are several limitations to our study. First, there were a limited number of patients managed with therapies other than anticoagulation. This could lead to our study to be underpowered to identify differences in practice patterns in the short timeframe chosen. Second, the retrospective nature of our study and the subjective activations of PERT makes it difficult to identify and investigate provider biases. Third, our institution's convention is to generally activate PERT for radiographic-proven PE. However, obtaining radiographic evidence in all suspected cases of PE during the pandemic was unfeasible. Furthermore, many patients with COVID19 were started empirically for PE based on D-dimer values alone. Many patients treated empirically for PE were not captured by this study, further undermining this study's ability to investigate provider biases. PERT utilization may have been helpful in cases of suspected PE to provide expert opinion regarding when to start empiric anticoagulation, when to transition to chemoprophylaxis after empiric treatment, and whether long-term anticoagulation or chemoprophylaxis is warranted at time of hospital discharge. In light of these limitations and the positive outcomes associated with PERT, we propose that other institutions consider changing their PERT guidelines to assist in cases of suspected PE (Fig. 1).

\section{Conclusions}

Patients with PE during COVID era were younger patients, had less malignancy, and developed clots later in their hospital course as compared to patients with PE in the pre-COVID era. During the COVID era, PERT activation correlated with reduction in LOS and trend towards fewer bleeding events. Medical management of PE changed during the COVID era for non-PERT patients and was associated with longer length of stay and a trend toward more bleeding. While our institutional utilization of PERT substantially decreased during the COVID era, PERT recommendations were unchanged and resulted in similar patient outcomes.

Author contributions BK and SBB are co-first authors. BK and SBB had full access to the data in the study take responsibility for the content of the manuscript, including integrity of the data and accuracy of the analyses. BK wrote the first draft of the manuscript; BK and SBB wrote subsequent drafts of the manuscript. $\mathrm{BK}$ and $\mathrm{BH}$ were involved in reviewing patient charts and collecting the data. SBB, NAE, RMG, $\mathrm{BH}, \mathrm{JMH}, \mathrm{CJ}, \mathrm{AKS}, \mathrm{DES}, \mathrm{EY}$, and TSM contributed to the study design, interpretation, and editing of the manuscript.

Funding None.

\section{Compliance with ethical standards}

Conflict of interest JMH receives funding from Penumbra, Inc and Inari Medical. BK, SBB, NEA, RMG, BH, CJ, AKS, DES, EY, and TSM have no conflicts of interest to disclose.

Ethical approval Institutional Review Board at New York University Langone Health Center approved this study (\#20-00501).

\section{References}

1. Tang N, Bai H, Chen X, Gong J, Li D, Sun Z (2020) Anticoagulant treatment is associated with decreased mortality in severe coronavirus disease 2019 patients with coagulopathy. J Thromb Haemost 18(5):1094-1099

2. Marginean A, Masic D, Brailovsky Y, Fareed J, Darki A (2020) Difficulties of managing submassive and massive pulmonary embolism in the era of COVID-19. JACC Case Rep 2:523

3. Bikdeli B, Madhavan MV, Jimenez D et al (2020) COVID-19 and thrombotic or thromboembolic disease: implications for prevention, antithrombotic therapy, and follow-up: JACC State-of-theArt Review. J Am Coll Cardiol 75(23):2950-2973

4. Schultz J, Giordano N, Zheng H et al (2019) EXPRESS: a multidisciplinary Pulmonary Embolism Response Team (PERT) - experience from a national multicenter consortium. Pulm Circ 9(3):2045894018824563

5. Rosovsky R, Zhao K, Sista A, Rivera-Lebron B, Kabrhel C (2019) Pulmonary embolism response teams: purpose, evidence for efficacy, and future research directions. Res Pract Thromb Haemost 3(3):315-330

6. Rosovsky R, Chang Y, Rosenfield K et al (2019) Changes in treatment and outcomes after creation of a pulmonary embolism response team (PERT), a 10-year analysis. J Thromb Thrombol 47(1):31-40

7. Chaudhury P, Gadre SK, Schneider E et al (2019) Impact of multidisciplinary Pulmonary Embolism Response Team availability on management and outcomes. Am J Cardiol 124(9):1465-1469

8. Konstantinides SV, Meyer G, Becattini C et al (2020) 2019 ESC Guidelines for the diagnosis and management of acute pulmonary embolism developed in collaboration with the European Respiratory Society (ERS). Eur Heart J 41(4):543-603

9. Panigada M, Bottino N, Tagliabue P et al (2020) Hypercoagulability of COVID-19 patients in Intensive Care Unit. A report of thromboelastography findings and other parameters of hemostasis. J Thromb Haemost 18:1738

10. Leonard-Lorant I, Delabranche X, Severac F et al (2020) Acute pulmonary embolism in COVID-19 patients on CT angiography and relationship to D-dimer levels. Radiology. https://doi.org/10.1148/ radiol.2020201561

11. Yuriditsky E, Horowitz JM, Merchan C et al (2020) Thromboelastography profiles of critically ill patients with coronavirus disease 2019. Crit Care Med 48:1319

12. Liu S, Luo P, Tang M et al (2020) Providing pharmacy services during the coronavirus pandemic. Int J Clin Pharm 42(2):299-304 
13. Badreldin HA, Atallah B (2020) Global drug shortages due to COVID-19: impact on patient care and mitigation strategies. Res Soc Adm Pharm. https://doi.org/10.1016/j.sapharm.2020.05.017

14. Kicska G, Litmanovich DE, Ordovas KG et al (2020) Statement from the North American Society for Cardiovascular Imaging on imaging strategies to reduce the scarcity of healthcare resources during the COVID-19 outbreak. Int J Cardiovasc Imaging. https:// doi.org/10.1007/s10554-020-01861-1

15. Sasangohar F, Jones SL, Masud FN, Vahidy FS, Kash BA (2020) Provider burnout and fatigue during the COVID-19 pandemic: lessons learned from a high-volume Intensive Care Unit. Anesth Analg 131(1):106-111

16. Singer BD, Jain M, Budinger GRS, Wunderink RG (2020) A Call for Rational Intensive Care in the era of COVID-19. Am J Respir Cell Mol Biol 63:132-133
17. Rice TW, Janz DR (2020) In defense of evidence-based medicine for the treatment of COVID-19 ARDS. Ann Am Thorac Soc 17:787-789

18. Gniadek TJ, Mallek J, Wright G et al (2020) Expansion of hospitalbased blood collections in the face of COVID-19 associated national blood shortage. Transfusion 60(7):1470-1475

Publisher's Note Springer Nature remains neutral with regard to jurisdictional claims in published maps and institutional affiliations. 